\title{
Comparison between Low/Programmable Freezing and Fast Freezing Protocols of Hungarian Guinea Fowl Semen
}

\author{
By Thieu Ngoc Lan Phuong* \\ Eva Varadi $^{+}$ \\ Barbara Vegit \\ Krisztina Liptoi ${ }^{+}$ \\ Judit Barna
}

\begin{abstract}
Semen cryopreservation is a practical method for banking germplasm from valuable indigenous species which have an increasing risk of extinction. Many scientific publications have described different protocols of avian sperm cryopreservation for both domesticated and non-domesticated avian species, involving cryoprotectant type and packaging method, as well as freezing and thawing rates. In this research, a comparative approach was used to evaluate two freezing protocols for guinea fowls: the modified slow programmable method using $10 \%$ ethylene glycol (EG), and the newly applied fast freezing method (pellet formation) with $6 \%$ dimethylacetamide (DMA). The efficiency of two protocols is measured by both in vitro sperm evaluation assay (determination of sperm concentration and motility, morphological and liveldead sperm analysis) and in vivo sperm evaluation assay (artificial insemination, determination of fertility rate and embryonic death). For fertility determination, candling of incubated eggs was used, extended by checking of the ratio of early embryonic mortality. In vitro qualification showed that the survival rate of live and intact spermatozoa was significantly higher after pelletation than after slow protocol (28.59\% vs 23.53\%, $p=0.022$, Mann-Whitney U test). In vivo qualification showed that artificial insemination of frozenthawed semen yielded $50.2 \%$ fertility rate with the pellet, while only $25.5 \%$ with the slow programmable method (while it was $84.8 \%$ in control, $p=0.027$, Kruskal-Wallis ANOVA test). The simple in vitro
\end{abstract}

\footnotetext{
*DVM, MSc, Research Centre for Farm Animal Gene Conservation (HáGK), Hungary.

${ }^{\dagger} \mathrm{MSc}$, Fellow Researcher, Research Centre for Farm Animal Gene Conservation (HáGK), Hungary.

${ }^{\ddagger} \mathrm{PhD}$, Fellow Researcher, Research Centre for Farm Animal Gene Conservation (HáGK), Hungary.

${ }^{+} \mathrm{PhD}$, Senior Researcher, Research Centre for Farm Animal Gene Conservation (HáGK), Hungary.

'DVM, PhD, Research Centre for Farm Animal Gene Conservation (HáGK), Hungary.
} 
Vol. 1, No. 3 Phuong et al.: Comparison between Low/Programmable Freezing...

examinations used in this research were not able to detect injuries which caused embryonic death during freezing procedure, therefore, despite the seemingly acceptable sperm surviving rate, the ratio of eggs containing a normal embryo in case of slow, programmable frozen-thawed semen artificial insemination was significantly lower than in case of fresh semen artificial insemination $15.88 \%$ and $70.46 \%$, while it was $28.37 \%$ in case of pellets, $p=0.038$, Kruskal

Wallis ANOVA test).

\section{Introduction}

Frozen storage of avian semen has been established for approximately more than 60 years (POLGE, 1951). Since the freezing tolerance of spermatozoa is the lowest among poultry species, due to their characteristic structure of sperm membrane (BLESBOIS et al., 2005), only domestic birds such as chicken, turkey, goose spermatozoa have been successfully frozen (DONOGHUE and WISHART, 2000). There are various methods available for avian cryopreservation such as vitrification in pellet form, rotation method, or slow, programmable freezing. Vitrification means the production of an amorphous state without any crystallization, defined by the viscosity. The amorphous state is like a "solid-liquid". The transformation is over a small temperature range described as the glass transition temperature. In simplified terms, it can be described as solidification of a liquid into an amorphous state while maintaining the same molecular orientations that existed in solution before the glass transition. The semen is frozen by rapid cooling through direct plunging of semen droplets into liquid nitrogen to form frozen pellets. The procedure takes a few seconds for cooling so removal of cryoprotectant before artificial insemination is not required. There were several modified pellet formations which have been described so far. In the study of Tereda, $0.2 \mathrm{ml}$ volumes of semen were dropped onto a depression in solid $\mathrm{CO}_{2}$, then, transferred into liquid nitrogen (TEREDA et al., 1989). In another study, Tsuletin dropped $0.2 \mathrm{ml}$ volumes of semen onto fluoro-plastic plate held in liquid nitrogen vapour at $-70^{\circ} \mathrm{C}$, then transferred into liquid nitrogen (TSELUTIN et al, 1995). And, recently, in the study of Iaffaldano, $60 \mu \mathrm{m}$ and $80 \mu \mathrm{m}$ volumes of semen were plunged into liquid nitrogen after equilibrating at $4 \mathrm{oC}$ for 1 to 5 minutes (IAFFALDANO et al., 2011). In the rotation method described by Kurbatov (1984), $1 \mathrm{ml}$ of samples within a $10 \mathrm{ml}$ capacity glass was rotated in liquid nitrogen vapour at $-70^{\circ} \mathrm{C}$ before plunging into liquid nitrogen. Contrary to these fast freezing methods, the slow freezing method using programmable freezers has been described by Lake in 1984, Wishart, Seigneurin and Blesbois in 1995. The samples within glass ampoules or plastic cryovials or straws are cooled from $5^{\circ} \mathrm{C}$ to $-35^{\circ} \mathrm{C}$ at between 1 to $10^{\circ} \mathrm{C} / \mathrm{min}$ before plunging into liquid nitrogen. Thawing systems involve placing the plunging samples from $-196^{\circ} \mathrm{C}$ into thermal bath or in a thermo-regulated hotplate adjusted to $60^{\circ} \mathrm{C}$ to $75^{\circ} \mathrm{C}$. There were a few studies that described the sperm freezing of guinea fowl so far (SEIGNEURIN and BLESBOIS, 2005; 
VÁRADI et al., 2013). In Hungary, successful application of semen freezing technology in guinea fowl has been carried out at the Research Centre for Farm Animal Gene Conservation (HáGK). Thus, this paper aims to describe in detail the fast and slow freezing of guinea fowl spermatozoa, and to compare these two protocols by using both in vitro and in vivo sperm evaluation assay.

\section{Materials and Methods}

30 one-year old Hungarian landrace guinea fowl males and 30 guinea fowl females originated from HáGK gene bank (SZALAY et al., 2009) were used as sperm donors and sperm receivers. The semen was collected by a non-invasive method, the dorso-abdominal massage technique, adapted from chicken semen collecting method (BURROWS AND QUINN, 1937) individually. A twoweek training of guinea cocks was carried out prior to the 8-week semen collection program.

\section{Slow, Programmable Protocol}

After collecting, pooled semen was diluted 1:3 with Lake's extender (LAKE, 1986) at room temperature. Then, $10 \%$ ethylene glycol (EG) was added into the diluted semen. $200 \mu \mathrm{l}$ from the samples were measured and put into cryovials. These cryovials were placed into the programmable freezing machine (Planer KRYO10). Cooling was started from $20^{\circ} \mathrm{C}$ with freezing rate $3^{\circ} \mathrm{C} / \mathrm{min}$. 25 minutes of equilibration time followed when the temperature reached $3^{\circ} \mathrm{C}$. Then, freezing rate $-1^{\circ} \mathrm{C} / \mathrm{min}$ was applied to cool the cryovials down to $-30^{\circ} \mathrm{C}$, and later freezing rate $-30^{\circ} \mathrm{C} / \mathrm{min}$ from $-30 \mathrm{oC}$ to $-60^{\circ} \mathrm{C}$. Finally, cryovials were removed from the freezing machine and plunged into liquid nitrogen. Composition of Lake's extender is demonstrated in table 3.

Vitrification in pellet form

After collecting, pooled semen was diluted 1:1 with Tselutin's extender (TSELUTIN et al, 1995) at room temperature. Then, the diluted semen was equilibrated for 20 minutes at $2^{\circ} \mathrm{C}$ and treated with cryoprotectant dimethylacetamide (DMA) 6\%. Treated semen was directly dropped into the liquid nitrogen $(25 \mu \mathrm{l} /$ pellet) with a pipette. The pellets were collected into cryovials and stored in liquid nitrogen.

Two freezing protocols used in this study were described in Table 1.

Table 1. Freezing Protocols

\begin{tabular}{|c|c|c|}
\hline Protocol & Slow, programmable & Pellet formation \\
\hline Type of cryocontainer & Cryovial $(200 \mu \mathrm{L})$ & Pellet $(25 \mu \mathrm{L})$ \\
\hline Diluents & Lake 's diluent $(1986)$ & Tselutin's diluent $(1995)$ \\
\hline Dilution rate & $1: 3$ & $1: 1$ \\
\hline Equilibration time & 25 mins at $3{ }^{\circ} \mathrm{C}$ & 20 mins at $2{ }^{\circ} \mathrm{C}$ \\
\hline Freezing rate & $-1^{\circ} \mathrm{C} / \mathrm{min}$ until $-30^{\circ} \mathrm{C}$ & $\begin{array}{c}\text { Dropped directly into } \\
\text { liquid nitrogen }\end{array}$ \\
\hline CPs & $-30^{\circ} \mathrm{C} / \mathrm{min}$ until $-60^{\circ} \mathrm{C}$ & $6 \%$ DMA \\
\hline
\end{tabular}


The samples frozen by slow, programmable method were thawed in a cooling cabin at $5^{\circ} \mathrm{C}$. The samples frozen by pellet method were thawed in an automatic machine at $70^{\circ} \mathrm{C}$.

The in vitro evaluation assay was carried out before freezing and after thawing on each occasion except for the motility and concentration parameter. Motility was examined only before freezing by subjective scoring from 0 to 5 based on the percentage of motile sperms and direction of sperm movement.

- Score 0: indicates no motility

- Score 1: indicates non-progressive motility (5-20\% of spermatozoa move)

- Score 2: indicates slow, progressive motility (20\%-40\% of spermatozoa move)

- Score 3: indicates side to side movement accompanied by slow, progressive motility ( $50 \%-70 \%$ of spermatozoa move)

- Score 4: indicates fast progressive motility $(75 \%-85 \%$ of spermatozoa move)

- Score 5: indicates very fast progressive motility $(85 \%-95 \%$ of spermatozoa move)

- Sperm concentration was measured by spectrophotometer (Accucell IMV, France) in million/ $\mu 1$ unit.

Morphological abnormalities and live/dead spermatozoa ratio of both fresh semen and frozen-thawed semen were determined by aniline blue-eosin staining.

For in vivo evaluation, 30 guinea hens were divided into 3 groups of 10 animals. Ten hens, used as controls, were inseminated with fresh semen twofold diluted in Lake's extender while the remaining guinea hens were inseminated with frozen-thawed semen, 10 hens with frozen-thawed semen produced by slow, programmable freezing and the other 10 hens by fast freezing in pellet form.

Artificial insemination was made 8 times per group totally, for 3 weeks, with an average frequency of 2 times/ week and an average inseminating dose of $290 \pm 52$ million spermatozoa/hen.

The collection of eggs started 2 days after the first insemination. Then, the eggs were incubated by an incubator and the fertility was checked by candling on day 7 or day 10 of incubation.

The germinal discs of eggs without an embryo were checked further by staining with PI. The eggs with embryonic death in the oviduct showed a stained nucleus under fluorescence microscope while the infertile eggs without a nucleus showed dark-red background without lighting points.

For statistical analysis, the Mann-Whitney U test and Kruskal Wallis ANOVA test of STATISTICA version 10 were used (STATSOFT INC., 2011) 


\section{Results}

\section{Results on in Vitro Evaluation}

The motility, concentration and morphological abnormalities of fresh semen collected for two freezing protocols are not significantly different $(p>0.05)$. The quality of frozen-thawed semen produced from slow, programmable freezing and fast freezing in pellet form was shown in Figure 1. After freezing and thawing, a significantly higher percentage of dead spermatozoa was detected in the fast freezing $(68.63 \%, \mathrm{p}<0.001)$. Interestingly, compared with fast freezing protocol, the slow, programmable freezing produced a significantly lower percentage of live, intact spermatozoa $(17.99 \%$ vs. $21.35 \%, \mathrm{p}=0.030$ ).

Figure 1. Comparison of Quality of Frozen-thawed Semen produced from Slow, Programmable Freezing and Fast Freezing in Pellet Form.

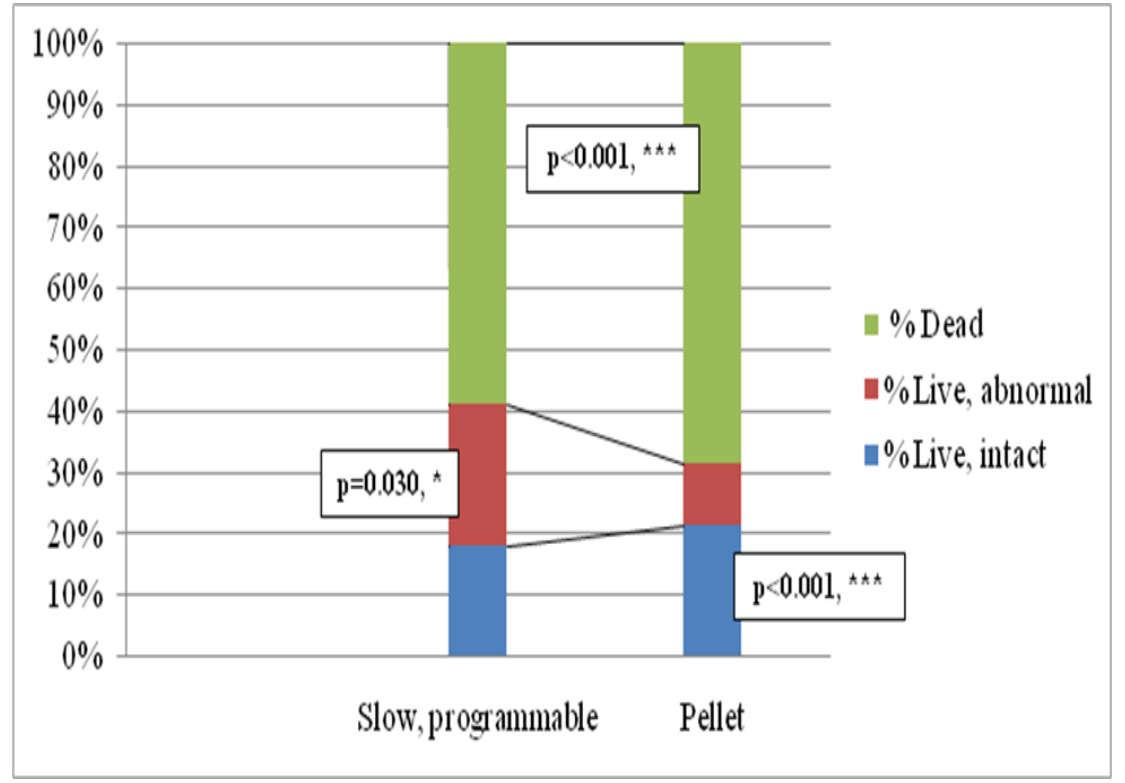

\section{Results on in Vivo Evaluation}

Fertility and embryo mortality in the three experimental groups following the artificial inseminations was shown in Figure 2. Artificial insemination with fresh semen yielded $84.8 \%$, while slow freezing and fast freezing protocol yielded $25.5 \%$ and $50.2 \%$ fertility rates, respectively. The percentage of infertile eggs in the case of insemination with frozen-thawed semen in pellet form was lower than in the case of insemination with slow, programmable frozen-thawed semen $(49.8 \%$ vs. $75.5 \%)$. The percentage of eggs with very early embryonic deaths in the oviduct were very high in the slow, programmable freezing group during the whole experimental period (week 1:18.42\%, week $2: 34.29 \%$, week $3: 23.53 \%$ ) while in the pellet freezing and control group, a decreasing tendency was observed (from $28.1 \%$ and $6.98 \%$ in the 1 st week to $4.5 \%$ and to $0 \%$ in 3rd week). Moreover, the percentage of eggs containing normal development in the slow, programmable group was 
Vol. 1, No. 3 Phuong et al.: Comparison between Low/Programmable Freezing...

extremely low compared with the pellet and control group (5.88\% vs. $28.37 \%$ vs. $70.46 \%, \mathrm{p}=0.038$ ).

Figure 2. Fertility and Embryo Mortality in the three Experimental Groups Following the Artificial Inseminations

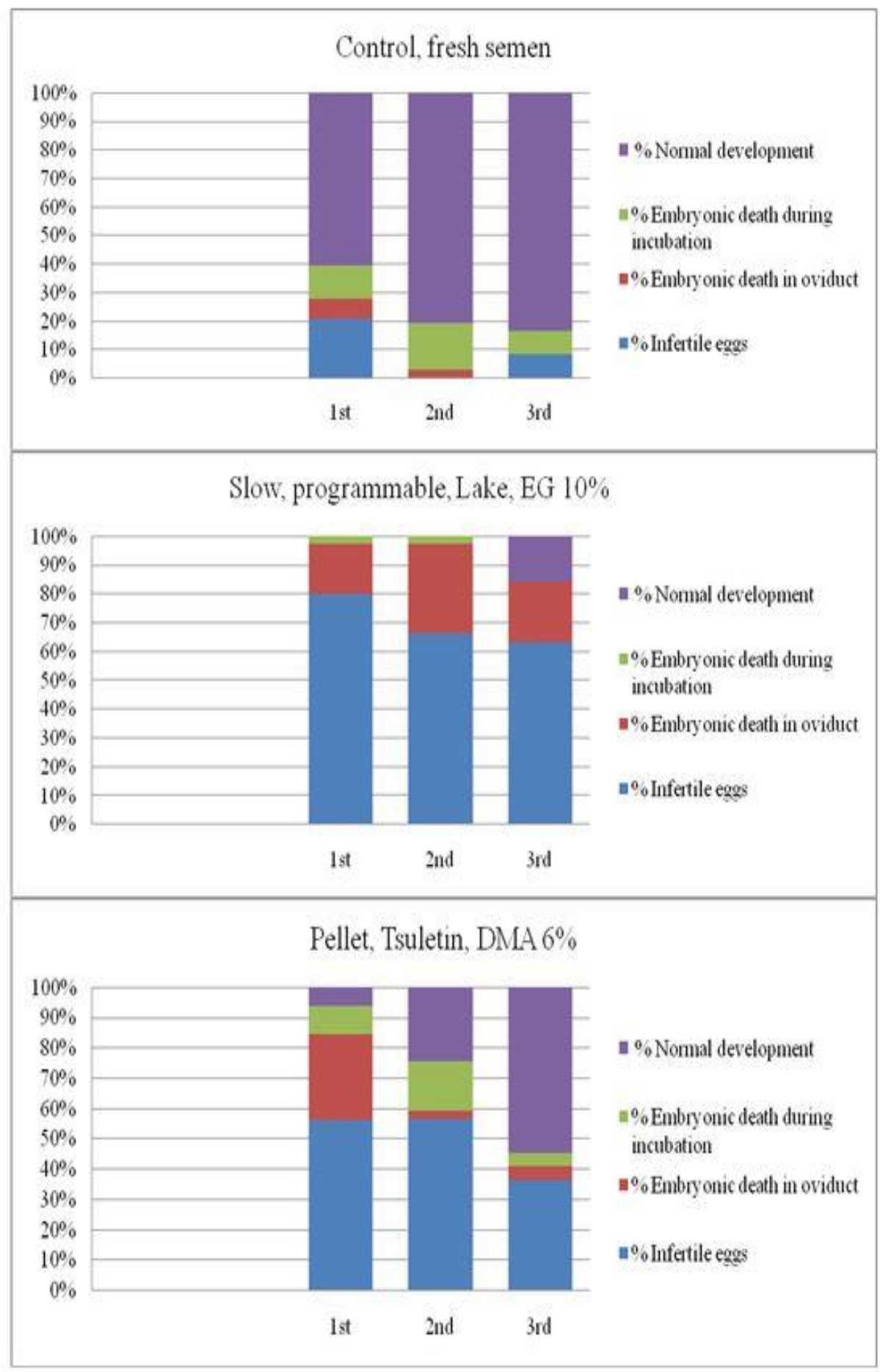




\section{Discussion}

In the present study, fresh semen of guinea fowls was successfully collected by the adapted dorso-abdominal massage technique from the chicken semen collecting method, and successfully frozen by both slow, programmable protocol with $10 \%$ EG and vitrification in pellet form with DMA 6\%. They produced an acceptable average sperm survival rate of live and intact spermatozoa (23.53\% and $28.59 \%)$. The highest sperm survival rate of live and intact spermatozoa, achieved in this study was $44.2 \%$ in case of the slow freezing and $36.49 \%$ in the pellet method. This result was absolutely lower than the highest sperm survival rate in previous study (higher than 60\%). A significantly higher percentage of live, abnormal spermatozoa was produced by slow, programmable freezing than that produced by vitrification $(23.06 \%$ vs. $10.02 \%, \mathrm{p}<0.001)$. However, the number of dead spermatozoa in pellet form was significantly higher than freezing by slow, programmable protocols $(68.63 \%$ vs. $58.96 \%, \mathrm{p}<0.001)$. Although the live, abnormal spermatozoa are able to fertilize after AI (VÉGI et al., 2005), the high rate of early embryonic death (a mean of $25.41 \%$ ) was observed in week 1 (18.42\%), $2(34.29 \%)$ and 3 $(23.53 \%)$ while in the case of the pellet group, a high rate of early embryonic death was observed only in the first week $(28.13 \%)$, then it dropped dramatically in the second week $(2.7 \%)$ and third week (4.5\%). This observation showed that the percentage of live, abnormal spermatozoa in frozen-thawed semen might give an effective estimate of eggs with embryonic death produced after AI. Even though, not only in-vitro but also in vivo tests were required for reliable testing of the effectiveness of freezing protocols applied in guinea fowl because the simple in-vitro examinations used in this study were not able to detect injuries such as DNA damage of spermatozoa during the freezing and thawing process. The in-vitro tests were necessary to predict the survival rate of spermatozoa and fertility rate in general and the invivo tests were inevitable to demonstrate the true quality of frozen-thawed guinea fowl semen. According to fertility results of the slow protocol, $75.5 \%$ of eggs were infertile, despite the seemingly acceptable sperm survival rate. Contrary to the slow freezing protocol, frozen-thawed semen produced by the pellet method produced $50.2 \%$ fertility rate after AI. Fertility rates of both slow, programmable and the pellet group achieved in this study were higher than the mean fertility rate obtained in the first study (20\%). Apart from the quality of AI semen expressed by the number of intact, viable spermatozoa remaining in post-thawing semen, it was observed that the increase of number of spermatozoa stored in the guinea hens oviduct by the 3rd weeks of AI, might be important in determining the fertility rate. The choice of cryoprotectant might be another factor that affected the fertilizing ability of frozen-thawed spermatozoa. Since, the fertility rates, $0 \%$ for EG and $8 \%$ for DMA, were reported in the first study, and $25.5 \%$ for EG, 50.2\% for DMA, the cryoprotectant DMA seemed to be less deleterious to sperm cells than EG. 
Vol. 1, No. 3 Phuong et al.: Comparison between Low/Programmable Freezing...

\section{Conclusion}

In this study, vitrification in pellet form with Tselutin extender and cryoprotectant DMA 6\% was proven to be another promising method for long term sperm storage of guinea fowl with $28.59 \%$ survival rate and $50.2 \%$ fertility. Although, the spermatozoa of guinea fowl are known to be very difficult to freeze, the highest survival rate $40.2 \%$, produced by slow freezing protocol and highest fertility rate, $63.64 \%$, produced by vitrification, has been observed from this research. It is difficult to compare results reported in the literature to the result obtained in this study due to variations in experimental designs such as differences in semen collecting schedule, semen dose, storage interval of semen, methods of freezing, and methods of evaluating frozen/thawed semen. Therefore, the concurrent comparison of semen freezing systems in guinea fowls should be carried out in further research studies. In order to establish an effective freezing protocol for guinea fowl semen with improved viability of spermatozoa during cryopreservation and fertilizing ability of frozen-thawed spermatozoa, the development of new sensitive invitro assays to evaluate sperm function may be valuable assessments.

\section{References}

Blesbois, E., Grasseau, I., Seigneurin, F. 2005: Membrane fluidity and the ability to survive cryopreservation in domestic bird spermatozoa. Reproduction, 129, p 371-378.

Burrows, W. H., Quinn, J. P. 1937: The collection of spermatozoa from the domestic fowl and turkey. Poultry Sci., 16, p 19-24.

Donoghue, A. M., Wishart, G. J. 2000: Storage of poultry semen. Animal Reproduction Science, 62, p 213-232.

Iaffaldano, N., Romagnoli, L., Manchisi, A., Rosato, M. P. 2011: Cryopreservation of turkey semen by the pellet method: Effects of variables such as the extender, cryoprotectants concentration, cooling time and warming temperature on sperm quality determined through principal components analysis. Theriogenology $76, \mathrm{p}$ 794-801.

Kurbatov, A. D., Narubina, L., Bubliaeva, G., Tsuletin, K.V. 1984: Cryopreservation of Cock Semen. Ptitsevodstvo, 11, P 28-29.

Lake, P. E., Ravie, O. 1984: An exploration of cryoprotective compounds for fowl spermatozoa. Poultry Science, 25, p 145-150.

Lake, P. E. 1986: The history and future of cryopreservation of avian germplasm. Poultry Science, 65, p 1-15.

Polge, C. 1951: Functional survival of fowl spermatozoa after freezing at $-70^{\circ} \mathrm{C}$. Nature, 167, p 949-950.

Seigneurin, F., Blesbois, E. 1995: Effect of freezing rate on viability and fertility of frozen-thawed fowl spermatozoa. Theriogenology, 43, p 1351-1358.

Seigneurin, F. and Blesbois, E. 2005: The first method of cryopreservation of guinea fowl semen. In: Proceedings of the French Poultry Research Days, Brittary, France. 
Szalay I., Dong Xuan, K.D.T, Virág G., Szentes, K.Á., Bódi, L. 2009: Prospects for conserving traditional poultry breeds of the Carpathian Basin. AWETH 5 (2), p 119-148.

Tereda, T., Ashizawa, K, Maeda, T., Tsusumi, Y. 1989: Efficacy of trehalose in cryopreservation of chicken. Jpn. J. Ani. Reprod., 35, p 20-25.

Tselutin, K., Narubina, L., Mavrodina, D., Tur, B. 1995: Cryopreservation of poultry semen. British Poultry Science, 36, p 805-811.

Varadi, E., Vegi, B., Liptoi, K., Barna, J. 2013: Methods for Cryopreservation of Guinea Fowl Sperm. PLoS ONE 8(4): e62759, DOI:10.1371/journal.pone. 0062759

Végi, B., Varga, Á., Szőke, Zs., Liptói, K., Várkonyi, E., Lennert L., Barna, J. 2005: Relationship Between Insemination of Frozen Sperm and Early Embryonic Death in Domestic Fowl. In: Proceedings of the 2nd Combined Workshop of Fundamental Physiology of the European Working Group of Physiology and Perinatal Development in Poultry, September 23-25, Berlin, p 55.

Wishart, G. J. 1995: Cryopreservation of avian spermatozoa. In: Cryopreservation and Freezing-Drying Protocols, Day, J.G., McLellan, M. R. (Eds),. Methods in Molecular Biology Humana Press, 38, p 167-177. 
